\title{
Remifentanil as Analgesia for Labor
}

\author{
Eliane C S Soares ${ }^{1}$, Michelle R Lucena ${ }^{2}$, Renata C Ribeiro ${ }^{3}$, Letícia L Rocha ${ }^{4}$, Walkíria W Vilas Boas, TSA ${ }^{5}$
}

Summary: Soares ECS, Lucena MR, Ribeiro RC, Rocha LL, Vilas Boas WW - Remifentanil as Analgesia for Labor.

Background and objectives: The neuraxial techniques currently represent the most effective methods for pain control during labor and the epidural block using ultradiluted anesthetic solutions is considered the gold standard promoting adequate pain relief with minimum side effects. In some situations however the use of these techniques is limited by the existence of maternal contraindications, or structural or material obstacles. In these cases, the alternatives are still precarious and scarce offering little optimistic results and of dubious effectiveness.

Content: This article presents through a literature review the available information on the use of remifentanil as an alternative technique for analgesia during labor discussing aspects of pharmacokinetics, analgesia efficacy, maternal satisfaction and maternal/fetal side effects.

Conclusions: The initial data show that remifentanil is a promising option to be employed in situations where the parturient cannot or does not want to receive the neuraxial analgesia.

Keywords: ANALGESIA, Opioids: remifentanil; ANALGESIA, Obstetrics.

[Rev Bras Anestesiol 2010;60(3): 334-346] @Elsevier Editora Ltda.

\section{INTRODUCTION}

The neuraxial techniques currently represent the most effective methods for pain control during labor ${ }^{1}$ and the epidural anesthesia using ultradiluted anesthetic solutions is considered the gold standard promoting adequate pain relief with minimal side effects ${ }^{2}$. These techniques however do not apply to all obstetric patients ${ }^{1,3,4}$. In some cases, they are impossible to be used due to absolute or relative maternal contraindications or by the unavailability of structure, material or adequate number of professionals trained at the institution. In addition to all these questions, there is the fact that the neuraxial techniques are not risk-free ${ }^{5}$. Important complications such as lumbar pain, post-dural puncture headache and neurological damage can appear due to the use of the technique, the medications and the materials employed. Immediate complications caused by the inadvertent injection of

Received from the Hospital Municipal Odilon Behrens, Belo Horizonte, MG

1. Anesthesiologist of the Hospitals Odilon Behrens and Vera Cruz; Coordinator of the Service of Obstetric Anesthesiology of the Hospital Universitário Risoleta Tolentino Neves, Belo Horizonte, MG

2. Anesthesiologist of the Hospitals Odilon Behrens and Socor

3. Anesthesiologist of the Hospital Odilon Behrens and Hospital das Clínicas da UFMG

4. ME3 of the CET/SBA of the IPSEMG

5. Anesthesiologist of the Hospital Lifecenter; PhD in Physiology at the Universidade Federal de Minas Gerais; Coordinator of the Anesthesiology Service of the Hospital Odilon Behrens; Responsible for the CET/SBA HC/UFMG

Submitted on October 6, 2009

Approved on February 10, 2010

Correspondence to:

Dra. Eliane Cristina de Souza Soares

Rua Sebastião Fabiano Dias, 233/2004A

Belvedere

30320-690 - Belo Horizonte, MG, Brasil

Email: elianecssoares@gmail.com anesthetic solutions in the subarachnoid, subdural and intravascular space can also occur at low incidences, but not negligible $\left(1: 2,900,1: 4,200\right.$ and $1: 5,000$, respectively) ${ }^{5}$. Such complications must not, in practice, represent a limitation to the use of neuraxial techniques, but in some situations they generate anxiety or even refusal on the part of the patient to accept the procedure.

In cases where it is not possible to perform the neuraxial analgesia the therapeutic options are scarce and precarious and are usually restricted to systemic analgesia with opioids or inhaled anesthetics (using mainly nitrous oxide). Such techniques however offer little optimistic results and some publications have raised serious doubts regarding its analgesic effectiveness ${ }^{6-8}$. Therefore, the development of an alternative technique to the neuraxial block for use during labor has been much sought after. This article aimed at assessing based on a literature review the role of remifentanil as an option for situations where the parturient cannot or does not want to receive the neuraxial analgesia.

\section{Use of opioids in labor analgesia}

The association between obstetric anesthesia and opioids throughout time has not been an easy one. The reports of sedation, nausea, vomiting, delayed gastric emptying and respiratory depression (in both the mother and the newborn) combined with a doubtful analgesic effectiveness has limited its use during labor ${ }^{4}$. An ideal intravenous opioid should result in adequate analgesia without interfering with the pattern of uterine contractions, fetal cardiography and its effect on the respiratory pattern of mother and fetus should be minimal so that the administration could be maintained until the end of the expulsion phase ${ }^{4}$. 
Among the currently employed opioids the meperidine is the most commonly used and studied. Most studies have shown that meperidine results in a certain degree of analgesia, but with inconsistent or insufficient response in a large number of cases, leading some authors to suggest that it acts more like a sedative than an analgesic agent 7,9 . The prolonged fetal depression associated with its use is a well-documented effect. It is caused by the drug's prolonged half-life ( 2.5 to 3 hours in the mother and 18 to 23 hours in the neonate) and the accumulation of active metabolites. Normeperidine, for instance, has a half-life of 60 hours and can be responsible for persistent neonatal respiratory depression for up to 3 to 5 days after the birth, even after low doses of meperidine ${ }^{7}$. In addition to these problems, meperidine affects the maternal gastric motility, modifies the cardiotocography and is related to fetal acidosis at the analysis of umbilical cord blood ${ }^{10}$.

Fentanyl, a highly liposoluble opioid with high proteinbinding capacity and 800 times more potent than meperidine, presents better analgesia performance with a peak of action 3 to 4 minutes after the infusion in bolus. However, fentanyl presents several adverse effects in the neonate, with a high incidence of naloxone at birth, low neurobehavioral scores up to 7 days after birth and an adverse dosedependent impact on the capacity to breastfeed soon after the birth ${ }^{7}$. The use of alfentanyl has also been assessed and the drug has shown worse analgesia results when compared to fentanyl, with lower neurobehavioral scores than the patients that received meperidine ${ }^{7}$. Sufentanyl is largely used in neuraxial analgesia with good results, but its venous use is very limited due to the potential effects of placental deposition and respiratory depression in the newborn ${ }^{7}$.

Therefore, none of the traditionally used and studied opioids presents the efficacy and pharmacokinetic profile that make them the ideal opioid for use during labor. Remifentanil, introduced in the market in the beginning of the 1990s, represents an actual possibility in the development of this alternative. For more than one decade, the properties of remifentanil have been explored in studies of surgical anesthesia, sedation and postoperative analgesia in non-obstetric populations ${ }^{11}$. Its use in obstetric anesthesia was only started in 1998, when a study established the pharmacokinetic profile of the drug in pregnant patients and neonates ${ }^{12}$. After this study, a series of case reports and clinical trials were performed and showed the potential of remifentanil to become a new option for the analgesia in labor.

\section{Remifentanil in obstetrics \\ Pharmacokinetics and pharmacodynamics}

Remifentanil is a synthetic opioid chemically related to fentanyl, which presents unique characteristics due to the presence of ester-type radicals in its chemical structure. It is a $\mu$ selective agonist, with $\mathrm{pKa}=7.07$ (weak base), little lipo- soluble and high protein-binding capacity $( \pm 70 \%$, mainly to alpha-1 acid glycoprotein) ${ }^{13}$. It is a fast-action drug ( \pm 60 seconds) and it is metabolized through hydrolysis by nonspecific plasma and tissue esterases, which results in rapid metabolism and ultra-short action (half-life sensitive to the context of 3 to 5 minutes) and prevents the accumulation of the drug after prolonged administration ${ }^{13,14}$. Its metabolites, all inactive, are eliminated in the urine and it is antagonized by naloxone ${ }^{14}$. Remifentanil crosses the placental barrier, but it is rapidly redistributed and metabolized by the neonate, who already has sufficient plasma and tissue esterases for its degradation ${ }^{12,14}$. The concentration ratio of remifentanil in the umbilical cord/uterine artery is 0.88 , showing that there is a fast exchange of maternal blood to the fetus, whereas the concentration ratios in umbilical artery and umbilical vein is 0.29 , suggesting significant metabolization and redistribution 4,14 . The clearance of remifentanil in pregnant patients seems to be two-fold higher than in non-pregnant patients, showing that the physiological alterations of pregnancy might be responsible for this difference due to the change in the volume of distribution, lower concentration of plasma proteins and increased nonspecific esterase activity $4,10,12$.

The administration of remifentanil is simple, being necessary to obtain a venous access and a continuous-infusion pump with IV-drip support and compatible syringes.

The main adverse effects observed in pregnant patients were nausea, vomiting, pruritus, sedation and respiratory depression 2,4,9-11,14-18.

The incidence of nausea and vomiting was variable being as high as $48 \%$ in one of the studies 4,9 . However, as these symptoms occur rather often during labor even without any analgesia it is necessary to estimate and distinguish the incidence directly associated with the use of opioids ${ }^{2,4,9}$. The association between the increase in the incidence of nausea and the use of remifentanil in the analgesia for labor was not statistically significant in previously published studies 2,14-17. In a randomized, controlled and double-blind study comparing remifentanil and epidural analgesia controlled by the patient the presence of nausea was observed in some patients before the start of the analgesic technique and the final results showed that, differently from what was expected, the incidence of nausea decreased after the start of the analgesia in the group that received remifentanil ${ }^{2}$.

Some studies have shown the occurrence of pruritus, normally of mild to moderate intensity and without report of the need for treatment 9,14 . There has been a report on the use of the drug in a patient that presented pruritus secondary to gestational cholestasis and in this case there was no increase in the symptom after the opioid was administered ${ }^{14}$. A preliminary study using patient-controlled analgesia (PCA) with remifentanil reported a patient with generalized pruritus, in whom the drug was discontinued ${ }^{14}$.

Sedation has been described in several cases, but it is always mild and rarely associated with apnea 4,10 . There have been very few reports of excessive sedation with the need for assisted ventilation ${ }^{18,19}$. Practically all studies that 
have been published showed episodes of alteration in the respiratory pattern and desaturation (without apnea and with $\mathrm{SpO}_{2}$ values > 90\%). These effects, however, were transient and easily corrected with the use of supplementary nasal oxygen and decrease in the dose used ${ }^{10}$. Due to such reports, however, remifentanil must be used in patients who are under constant follow-up and continuous monitoring of oxygen saturation and the professional must have at his/her disposal a supplementary source of oxygen for use in nasal catheter or facial mask and adequate equipment for assisted ventilation and resuscitation $2,4,10,11,14-$ 17.

There has been no evidence of hemodynamic instability with the use of remifentanil ${ }^{14}$. The development of acute tolerance has been observed with several opioids. However, studies in volunteers and observation of patients in intensive care maintained with a continuous infusion of remifentanil (to aid mechanical ventilation) have not shown the occurrence of acute tolerance ${ }^{20,21}$.

Some authors studied the effects of the different opioids (including remifentanil) and local anesthetics in the isolated uterine muscle of pregnant rats ${ }^{10}$. The results showed that these two groups of medications reduce the contractility of the uterine muscle fiber. Such medications, when used through epidural or spinal anesthesia, do not reach sufficient plasma concentrations to induce the effects described above, but when they are used intravenously, they can reach the concentrations used in the experiments ${ }^{10}$. However, there have been no clinical studies that showed in vivo results that were similar to the ones observed in vitro.

There has been no association between the infusion of remifentanil and any deterioration in cardiotocography that would indicate intervention or investigation, as well as in the fetal blood samples. A transient variability in the tracing has been noticed, but these effects are much less frequent than the ones observed with the systemic administration of other opioids 2,11,14,15. The Apgar scores and blood $\mathrm{pH}$ of the umbilical cord did not present abnormal results $2,9,12,14$. None of the studies in analgesia demonstrated the need for neonatal use of naloxone or an unexpectedly low Apgar score (without other reasons involved in it), which leads us to conclude that the dose used in the analgesia is rapidly metabolized also by the neonate ${ }^{4,11}$. In the reports in which naloxone was necessary for neonatal resuscitation, remifentanil was used to supplement the general anesthesia in infusions $>0.1 \mu \mathrm{g} \cdot \mathrm{kg}^{-1} \cdot \mathrm{min}^{-1}$ and associated to other drugs 4 . The pharmacokinetic profile of remifentanil in neonates is similar to that of older children and adults ${ }^{12}$, and remifentanil is used in the neonatal ICU in patients undergoing mechanical ventilation and surfactant administration ${ }^{22}$. Remifentanil does not bind to NMDA and GABA receptors and therefore it is not associated with apoptotic neurodegeneration and persistent deficits of memory and learning by the newborn when used in pregnant patients ${ }^{3}$.

\section{Remifentanil in analgesia for labor effectiveness and maternal satisfaction}

The first reports on the use of remifentanil for analgesia during labor involved pregnant patients that presented some type of absolute contraindication to neuraxial analgesia and agreed to receive an alternative analgesic technique ${ }^{24}$. The first study that reported the use of the drug in healthy patients was published in the year $2000^{25}$. The pharmacokinetic profile of remifentanil suggested the possibility of its use in bolus, thus matching the peak of the action with the peak of the pain produced by the uterine contractions. In this study, the drug was administered to four parturients as manual bolus at the start of the contractions perceived by the uterine dynamics. The results showed that there was a delay in the start of the action in relation to the contractions and the peak of the analgesic action occurred during the interval between them. The final conclusion was that remifentanil was not effective as an analgesic technique for labor ${ }^{25}$. Computerized simulations of the concentration in an effector site had already predicted a half-life of equilibrium (blood-site effector) of 1.3-1.6 minutes and a study that analyzed the effect on the ventilator pattern in healthy volunteers with a bolus of $0.5 \mu \mathrm{g} . \mathrm{kg}^{-1}$ showed that the start of the effect occurred in 30 seconds with a peak in 2.5 minutes. These data suggested that the bolus infusion in the beginning of the uterine dynamics would make it difficult to match to the peak effect with the peak of the pain ${ }^{4}$. In 2001, Blair et al. ${ }^{9}$ investigated the effectiveness and safety of PCA with remifentanil in 21 parturients. The drug was used in an initial bolus of $0.25 \mu \mathrm{g} \cdot \mathrm{kg}^{-1}$, with a minimum interval (lockout time) of 2 minutes, gradual increase up to a maximum dose of $1 \mu \mathrm{g} \cdot \mathrm{kg}^{-1}$ and in association or not with a continuous infusion of 0.025 to $0.05 \mu \mathrm{g} \cdot \mathrm{kg}^{-1} \cdot \mathrm{min}^{-1}$. There was a considerable decrease in the pain score in $90 \%$ of the patients, without the need to administrate an associated continuous infusion. There were no significant decreases in the fetal cardiography and the Apgar scores and blood $\mathrm{pH}$ values of the umbilical cord remained within normal ranges. The authors concluded, then, that the technique was safe and resulted in analgesia that in spite of being incomplete was acceptable during labor.

In a case report published in 2002, Owen et al. ${ }^{26}$ reported the use of remifentanil in a morbidly obese patient with antiphospholipid syndrome, severe thrombocytopenia, kidney failure, preeclampsia and epigastric pain (with normal liver function and abdominal ultrasound results). Remifentanil was used as a continuous infusion at doses that varied from $0.05 \mu \mathrm{g} \cdot \mathrm{kg}^{-1} \cdot \mathrm{min}^{-1}$ and $0.2 \mu \mathrm{g} \cdot \mathrm{kg}^{-1} \cdot \mathrm{min}^{-1}$. This case report stood apart from others due to fact that the infusion was maintained for 34 consecutive hours. The mean pain score obtained was 4 in 10 and although the total pain control (score $<2$ in 10) was not obtained, the researchers reported that the patient, family members and obstetric team were satisfied with the degree of analgesia attained. 
In the same year, Volmanen et al. ${ }^{15}$ used remifentanil as PCA infusion for a period of 60 minutes while evaluating 17 healthy pregnant patients. In this study, an initial bolus of $0.2 \mu \mathrm{g} . \mathrm{kg}^{-1}$ was used (lockout of 1 minute), which was administered as soon as the patient perceived the first signs of uterine contraction, with an increase of $0.2 \mu \mathrm{g} . \mathrm{kg}^{-1}$ per dose when the analgesia was ineffective (up to a maximum of $0.8 \mu \mathrm{g} . \mathrm{kg}^{-1}$ ). All patients reported adequate pain relief (scores varying from 3.1 to 5.2 in a scale of 10 ), with a mean bolus of $0.4 \mu \mathrm{g} . \mathrm{kg}^{-1}$ and a maximum bolus of $0.8 \mu \mathrm{g} . \mathrm{kg}^{-1}$.

In 2005, Evron et al. ${ }^{17}$ compared in a double-blind study PCA with remifentanil with intravenous meperidine in 88 parturients ASA I and II who did not wish initially to receive neuraxial analgesia. The dose in bolus of remifentanil was initially $20 \mu \mathrm{g}$ (lockout of 3 minutes), with increases of 5 $\mu \mathrm{g} . \mathrm{kg}^{-1}$ per dose in case of insufficient analgesia up to a maximum of $1,500 \mu \mathrm{g}$. hour ${ }^{-1}$. Meperidine was given as an initial dose of $75 \mathrm{mg}$ per patient, with additional doses of $50 \mathrm{mg}$ in case of insufficient analgesia up to a maximum of $200 \mathrm{mg}$. The doses of remifentanil ranged from 0.27 to $0.93 \mu \mathrm{g} . \mathrm{kg}^{-1}$ per dose and were more effective than meperidine in the control of pain, with higher scores of maternal satisfaction and lower sedative effect. Blair et al. ${ }^{27}$ also compared PCA with remifentanil (at doses of $40 \mu \mathrm{g} . \mathrm{kg}^{-1}$ bolus with 2-minute intervals) with PCA using meperidine (5 $\mathrm{mg}$ with a minimum interval of 10 minutes). The pain scores were similar for both drugs, but the level of satisfaction with the analgesia was higher in the group that used remifentanil.

Volmanen et al. ${ }^{16}$ conducted another randomized and double-blind study in 2005 using PCA with remifentanil, this time compared to nitrous oxide. In this study, 15 patients alternated remifentanil and nitrous for periods of $20 \mathrm{mi}-$ nutes with a wash-out of 20 minutes between techniques. Remifentanil was used as PCA in bolus of $0.4 \mu \mathrm{g} . \mathrm{kg}^{-1}$ (lockout of 1 minute) with consecutive increases (without a ceiling dose) and the nitrous oxide was offered through a facial mask at a concentration of $50 \%$. Both medications were started as soon as the patient perceived the first sign of uterine contraction. The decrease in the pain score was higher during the use of remifentanil, when compared to the nitrous oxide $(1.5 \times 0.5$ in a scale of 10 , with $p<0.001)$, and 14 of the 15 patients chose remifentanil as the best analgesic technique.

Volikas et al. ${ }^{14}$ investigated the use of remifentanil by PCA in 50 parturients in an observational prospective study. A dose in bolus of $0.5 \mu \mathrm{g} \cdot \mathrm{kg}^{-1}$ (lockout of 2 minutes) was used and the results showed mean pain scores of $46 \mathrm{~mm}$ (in $100 \mathrm{~mm}$ ). This study investigated the plasma concentrations of remifentanil in the mother and the blood of the umbilical cord and the data obtained confirmed previous results of transplacental crossing and fetal metabolism. In 2007, Balki et al. ${ }^{19}$ compared two regimens of remifentanil use in bolus by PCA associated with continuous infusion.
Both groups started analgesia with a continuous infusion of $0.025 \mu \mathrm{g} \cdot \mathrm{kg}^{-1} \cdot \mathrm{min}^{-1}$ associated to a bolus by PCA of 0.25 $\mu \mathrm{g} . \mathrm{kg}^{-1}$ (lockout of 2 minutes). In the first group, the rate of continuous infusion was maintained constant and the bolus was increased when necessary by $0.25 \mu \mathrm{g} \cdot \mathrm{kg}^{-1}$ up to a maximum of $1 \mu \mathrm{g} \cdot \mathrm{kg}^{-1}$. In the second group the bolus dose was fixed at $0.25 \mu \mathrm{g} . \mathrm{kg}^{-1}$ and the rate of continuous infusion was increased when necessary by $0.025 \mu \mathrm{g} \cdot \mathrm{kg}^{-1} \cdot \mathrm{min}^{-1}$ up to a maximum of $0.1 \mu \mathrm{g} \cdot \mathrm{kg}^{-1} \cdot \mathrm{min}^{-1}$. The results showed mean pain and maternal satisfaction scores that were equivalent in the two groups, with a higher incidence of side effects in the second group.

Recently, Volmanen et al. ${ }^{2}$ compared the epidural analgesia with PCA with remifentanil. The study was randomized, double-blind and included 45 patients. The epidural analgesia was performed with a bolus of $10 \mathrm{~mL}$ of levobupivacaine solution of $0.625 \mathrm{mg} \cdot \mathrm{mL}^{-1}$ associated to PCA with fentanyl $2 \mu \mathrm{g} \cdot \mathrm{kg}^{-1}$, and remifentanil was given in bolus of 0.3 with progressive increase up to maximum dose of 0.7 $\mu \mathrm{g} . \mathrm{kg}^{-1}$, also as PCA. The data obtained showed that the epidural anesthesia presented lower pain scores (means of 5.2 for the epidural anesthesia $\times 7.3$ for remifentanil), but the evaluation of pain relief (in a scale of 0 to 4 ) showed a similar result in both groups. The increase in the threshold of tolerance to pain was discussed in this study and it is a phenomenon that has been observed and described with the use of opioids in several situations. It seems to be caused by the association of sedation, euphoria and well-being connected with the use of these drugs and, during the analgesia for labor, it seems to be responsible for increasing the satisfaction levels even when the scores of pain are less adequate ${ }^{2,16}$

Table I shows a summary of the main studies conducted with their respective doses, mean decrease in pains cores and incidence of conversion to epidural anesthesia. As shown in the table, some of these studies evaluated the rate of conversion to neuraxial analgesia due to the patient's request and the authors used this information as one of the parameters for the assessment of maternal satisfaction. The values obtained from all studies were between 5 and $10.8 \%$ except for the study by Thurlow which presented a rate of conversion of $38 \%$ 4,11 . In the study by Volmanen ${ }^{2}, 86 \%$ of the patients receiving remifentanil chose to maintain this technique throughout the labor.

An important aspect to be discussed is that the analgesia represents only one of the components of maternal satisfaction during the labor experience and that complete analgesia (pain score $<2$ in 10) is not among the most important aspects when the patient rates the level of satisfaction. A review of 137 studies involving more than 14,000 parturients in different countries showed that personal expectations, good relation with/trust in the obstetric team, constant follow-up, adequate support and possibility of making choices and consenting with decisions were the factors that had the highest 
Table I - Use of Remifentanil (PCA Technique) during Labor

\begin{tabular}{|c|c|c|c|c|}
\hline Study & $\begin{array}{l}\text { Dose in bolus } \\
\mu \mathrm{g} \cdot \mathrm{kg}^{-1} \text {.dose }\end{array}$ & $\begin{array}{l}\text { Lockout } \\
\text { time }\end{array}$ & $\begin{array}{l}\text { Pain score } \\
\text { (in } 100 \mathrm{~mm} \text { ) }\end{array}$ & $\begin{array}{l}\text { Patients that chose conversion } \\
\text { to epidural analgesia }\end{array}$ \\
\hline Blair (2001) ${ }^{9}$ & $0.25-0.5$ & $2 \min$ & Mean $=50 \mathrm{~mm}$ & 2 in $21(9.5 \%)$ \\
\hline Thurlow (2002) 28 & 0.20 & $2 \min$ & Mean $=48 \mathrm{~mm}$ & 7 in $18(38 \%)$ \\
\hline Volmanen (2005) ${ }^{16}$ & 0.4 & $1 \mathrm{~min}$ & Decrease of $15 \mathrm{~mm}$ & - \\
\hline Blair (2005) 27 & $40 \mu \mathrm{g}$ (fixed) & $2 \min$ & Mean $=64 \mathrm{~mm}$ & 2 in $20(10 \%)$ \\
\hline Volmanen (2002) ${ }^{15}$ & $0.2-0.8$ & $1 \mathrm{~min}$ & Decrease of $42 \mathrm{~mm}$ & - \\
\hline Volikas (2005) ${ }^{14}$ & 0.5 & $2 \min$ & Mean $=46 \mathrm{~mm}$ & 5 in $50(10 \%)$ \\
\hline Balki (2007) ${ }^{19}$ & $0.25+\mathrm{Cl}$ & $2 \min$ & No information & 1 in $20(5 \%)$ \\
\hline Volmanen (2008) ${ }^{2}$ & $0.1-0.9$ & $1 \mathrm{~min}$ & Mean $=73 \mathrm{~mm}$ & - \\
\hline
\end{tabular}

$\mathrm{Cl}-$ continuous infusion

Modified from Hill D et al. - The use of remifentanil in Obstetrics. Anesthesiol Clin, 2008;26:169-82.

influence on satisfaction ${ }^{29}$. The lack of satisfaction when related to pain is associated to the unavailability or delay in the moment of analgesia introduction, either neuraxial or alternative ${ }^{22}$. Therefore, even if remifentanil is not capable of eliminating all the pain-related discomfort associated to the contractions it represents a good alternative to epidural analgesia if the maternity ward can offer the key elements described in the conduction of the labor procedure.

In the last study, published until the end of this review article, an Italian group ${ }^{30}$ presented the use of remifentanil in continuous infusion without bolus with an initial dose of $0.025 \mu \mathrm{g} \cdot \mathrm{kg}^{-1} \cdot \mathrm{min}^{-1}$ and gradual increases every 3 minutes until adequate analgesia was achieved or a maximum dose of $0.15 \mu \mathrm{g} \cdot \mathrm{kg}^{-1} \cdot \mathrm{min}^{-1}$ was used. The study was conducted in a maternity ward in which neuraxial analgesia is not routinely available. The mean score of pain 30 minutes after the infusion was $3.6 \pm 1.5$ in 10 , and $93 \%$ of the patients were satisfied with the received analgesia ${ }^{30}$.

Our group is currently conducting two clinical trials using intravenous remifentanil as analgesic technique for labor as a continuous infusion, without bolus, with initial doses of 0.025 $\mu \mathrm{g} \cdot \mathrm{kg}^{-1} \cdot \mathrm{min}^{-1}$ and a maximum dose of $0.15 \mu \mathrm{g} \cdot \mathrm{kg}^{-1} \cdot \mathrm{min}^{-1}$. Our preliminary results are extremely promising, mainly at the first stage, and we did not observe relevant maternal or fetal side effects. We have, at this time, incorporated this technique as the choice for patients who present contraindication to the neuraxial block.

\section{Controversies and perspectives}

The information presented here show that remifentanil has the potential to occupy an alternative position as analgesic technique during labor. Some questions discussed here, however, deserve attention and remain controversial with the data that have been published to date.

The first one concerns the infusion regimen used. In the current context, several forms of infusion and doses have been studies and there is no consensus regarding which one is more effective and safe. From the pharmacokinetic point of view, remifentanil has a profile that seems to be more compatible with the PCA form of administration. In the studies that have performed, the doses in bolus ranged from 0.2 to $0.93 \mu \mathrm{g} \cdot \mathrm{kg}^{-111}$. This type of infusion, however, tends to result in a higher incidence of side effects, coinciding with the peak of action of the bolus and requires skill from the patient to synchronize this peak of analgesia with the contractions. The continuous infusion at low doses also seems to be an adequate alternative associated or not to doses in bolus, but studies employing this type of regimen are still scarce, although they have shown promising results.

Therefore, additional studies are necessary to establish the most adequate, efficient and safe way to employ remifentanil. However, it is important to remember that historically many controlled studies were necessary along the years to determine the adequate doses for the currently employed neuraxial techniques.

Remifentanil crosses the placental barrier easily and for that reason it can have potent and prompt effects on the fetus and the newborn. The descriptions of the use of remifentanil in anesthesia for in utero surgeries (EXIT) and Cesarean sections using higher doses than the ones used for analgesia, showed the occurrence of fetal immobility and loss of variability of fetal heartbeats ${ }^{10}$. However, studies to carefully assess these effects in the fetus and neonate with the doses used in analgesia, using continuous monitoring of cardiotocography and arterial blood gases of blood from the umbilical cord are still necessary.

Regarding the costs, there were no data in literature comparing the costs of analgesia using remifentanil with the other techniques. An analysis performed in our institution showed no significant difference between the costs with the epidural technique (considering the use of the catheter needle, anesthetic solutions and material sterilization) when compared to analgesia with remifentanil (considering 
loaned infusion pumps, supports, extensors and mean cost of the medication).

As it occurs with some drugs routinely employed in pregnant patients (such as fentanyl by spinal administration) remifentanil has not been released for use during pregnancy. This isolated fact does not represent an obstacle to its use, but the number of studies and patients that received this medication is still limited to justify the routine use of the technique.

\section{CONCLUSION}

Remifentanil as continuous infusion at a maximum dose of $0.15 \mu \mathrm{g} \cdot \mathrm{kg}^{-1} \cdot \mathrm{min}^{-1}$ associated or not to a bolus administered by PCA seems to result in an acceptable and clinically satisfactory mean decrease in pain scores during labor, apparently without interfering with its evolution. The described side effects are minimal and easily reversible in the mother and newborn and the technique does not affects the parturient's capacity to experience the act of delivering. Although the available data do not allow the substitution of the neuraxial techniques by remifentanil, the drug is probably one of the best currently available alternatives for patients that for some reason do not wish to or cannot have a neuraxial block. In these cases, its better use is during analgesia for labor. However, the number of populations and studied patients is still low and further studies are necessary to confirm the initial data, to determine the most adequate doses and infusion regimens, cost assessment and maternal/fetal safety. 